Anaesthesist 2022 $\cdot 71: 510-517$

https://doi.org/10.1007/s00101-021-01074-8

Eingegangen: 27. Juli 2021

Überarbeitet: 29. September 2021

Angenommen: 2. November 2021

Online publiziert: 26 . November 2021

○ Der/die Autor(en) 2021

\section{Umsetzung anästhesiologischer Fast-Track-Maßnahmen bei kolorektalen Resektionen}

\author{
Eine Umfrage unter Mitgliedern der DGAI
}

\author{
M. A. Willis ${ }^{1} \cdot$ M. Thudium ${ }^{2} \cdot$ C. J. van Beekum ${ }^{1} \cdot$ M. Söhle ${ }^{2} \cdot$ M. Coburn ${ }^{2} \cdot$ J. C. Kalff ${ }^{1}$. \\ T. O. Vilz' \\ ${ }^{1}$ Klinik- und Poliklinik für Allgemein-, Viszeral-, Thorax- und Gefäßchirurgie, Universitätsklinikum Bonn, \\ Bonn, Deutschland \\ ${ }^{2}$ Klinik für Anästhesiologie und Operative Intensivmedizin, Universitätsklinikum Bonn, Bonn, Deutschland
}

\title{
Zusammenfassung
}

Einleitung: Beim Fast-Track(FT)-Konzept handelt es sich um multimodale, interdisziplinäre Maßnahmen zur perioperativen Patientenbetreuung zur Reduktion postoperativer Komplikationen. Trotz guter Evidenz erscheint die Umsetzung verbesserungswürdig, wobei sich nahezu alle Untersuchungen ungeachtet des interdisziplinären Aspektes auf die Implementierung der chirurgischen Module fokussieren. Die Adhärenz zu den anästhesiologischen Maßnahmen (präoperative Patientenoptimierung, Prämedikation, Volumen- und Wärmemanagement, Schmerztherapie) ist hingegen nur unzureichend untersucht. Um den Status quo zu erfassen, wurde eine Umfrage zur Umsetzung der anästhesiologischen FT-Bausteine unter den Mitgliedern der Deutschen Gesellschaft für Anästhesiologie und Intensivmedizin (DGAI) durchgeführt und analysiert, wo mögliches Verbesserungspotenzial besteht.

Methoden: Mithilfe des Online-Umfragetools SurveyMonkey ${ }^{\circledR}$ wurden 28 Fragen zur perioperativen anästhesiologischen Versorgung kolorektalchirurgischer Patienten an die Mitglieder der DGAI verschickt und die Adhärenz zu den FT-Maßnahmen analysiert. Ergebnisse: Während einige der FT-Maßnahmen (Wärmemanagement, PONVProphylaxe) bereits routinemäßig umgesetzt sind, gibt es bei anderen Bausteinen eine Divergenz zwischen den aktuellen Empfehlungen und der klinischen Umsetzung. Besonders betroffen sind neben der Prämedikation insbesondere interdisziplinäre Maßnahmen (Prähabilitation) sowie Maßnahmen, die mehrere Schnittstellen (OP, Aufwachraum (AWR), Station) betreffen, wie beispielsweise Volumenmanagement oder perioperative Schmerztherapie

Fazit: Die anästhesiologischen Empfehlungen des FT-Konzepts werden in Deutschland nur teilweise umgesetzt. Hiervon sind insbesondere die interdisziplinären Bausteine sowie Maßnahmen an den Schnittstellen OP-AWR-Station betroffen. Die Bildung eines interdisziplinären FT-Teams und die interdisziplinäre Ausarbeitung von SOP können die Adhärenz optimieren, was wiederum das kurz- und langfristige Outcome der Patienten verbessert.

\section{Schlüsselwörter}

Versorgungsforschung · ERAS · Perioperative Medizin · Evidenzbasierte Medizin · Compliance

Bereits 1995 konnte Kehlet durch die Implementierung des interdisziplinären Fast-Track(FT)-Konzepts eine reduzierte Komplikationsrate mit verkürzter Krankenhausverweildauer nach kolorektalen Resektionen erreichen. Dieses Konzept wurde seitdem weiterentwickelt; neben einer geringeren Komplikationsrate zeigen sich in neuen Untersuchungen eine positive Beeinflussung der perioperativen Immunantwort sowie ein verlängertes 5-Jahres-Überleben nach onkologi- 


\begin{tabular}{|c|c|c|}
\hline \multicolumn{2}{|c|}{ Umfrageteilnehmer } & \multirow{2}{*}{$\begin{array}{l}\text { Prozent } \\
2,3(n=10)\end{array}$} \\
\hline Alter & $<30$ Jahre & \\
\hline$(n=434)$ & 31-40 Jahre & $24,9(n=108)$ \\
\hline & 41-50 Jahre & $27,7(n=120)$ \\
\hline & 51-60 Jahre & $33,4(n=145)$ \\
\hline & 61-70 Jahre & $11,8(n=51)$ \\
\hline \multirow{7}{*}{$\begin{array}{l}\text { Berufliche } \\
\text { Position } \\
(n=433)\end{array}$} & Assistenzarzt & $12,5(n=10)$ \\
\hline & Facharzt & $26,1(n=113)$ \\
\hline & Oberarzt & $33,0(n=143)$ \\
\hline & Leitender Oberarzt & $9,5(n=41)$ \\
\hline & Chefarzt & $18,0(n=78)$ \\
\hline & Niedergelassen & $0,5(n=2)$ \\
\hline & Sonstiges & $0,5(n=2)$ \\
\hline \multirow{6}{*}{$\begin{array}{l}\text { Arbeitgeber } \\
(n=434)\end{array}$} & Universitätsklinik & $15,0(n=65)$ \\
\hline & Krankenhaus der Maximalversorgung & $14,1(n=61)$ \\
\hline & Krankenhaus der Schwerpunktversorgung & $31,1(n=135)$ \\
\hline & Krankenhaus der Grund- und Regelversorgung & $38,3(n=166)$ \\
\hline & Einzelpraxis/Gemeinschaftspraxis/Praxisgemeinschaft & $0,2(n=1)$ \\
\hline & Sonstiges & $1,4(n=6)$ \\
\hline
\end{tabular}

scher Kolorektalchirurgie. Da eine hohe Adhärenz die Voraussetzung für die positiven Effekte ist, muss eine flächendeckende Umsetzung des FT-Konzepts erfolgen.

\section{Hintergrund und Fragestellung}

Das inzwischen gut bekannte FT-Konzept zielt darauf ab, durch verschiedene perioperative Maßnahmen die Rekonvaleszenz zu beschleunigen und unerwünschte Folgen einer Operation, wie beispielsweise infektiöse Komplikationen oder postoperative Darmmotilitätsstörungen, abzumildern oder zu verhindern. Neben Empfehlungen zu klassisch chirurgischen Aspekten, wie beispielsweise der Operationstechnik, der Anlage von Drainagen oder dem postoperativen Kostaufbau, beinhaltet das FT-Konzept auch viele Empfehlungen für die interdisziplinäre und anästhesiologische Betreuung der Patienten. Dazu gehören u.a. Empfehlungen zur präoperativen Patientenoptimierung/Prähabilitation, zur Prämedikation, zum perioperativen Volumen- und Wärmemanagement sowie zur intra- und postoperativen Schmerztherapie [6].

Die Wirksamkeit des FT-Konzepts, das seit der Erstbeschreibung durch Kehlet kontinuierlich weiterentwickelt wurde, konnte bereits in verschiedenen Meta- analysen nachgewiesen werden. Es hat sich jedoch gezeigt, dass zur Erzielung des gewünschten Effekts eine Adhärenz zu den Maßnahmen von mehr als 70\% erforderlich ist [5]. Wird dies erreicht, so resultiert neben einer Reduktion der Komplikationsrate und einer Verkürzung der Krankenhausverweildauer (KVD) auch ein verbessertes onkologisches Outcome nach der Resektion kolorektaler Karzinome [10]. Jedoch spielt nicht nur eine stringente Umsetzung des Gesamtkonzepts eine wichtige Rolle, selbst Einzelmaßnahmen können entscheidend das kurz- und langfristige Outcome verbessern. Dies konnte insbesondere für die intraoperative Volumentherapie gezeigt werden: Aktuelle Metaanalysen haben beispielsweise eine Reduktion der Komplikationsrate sowie eine schnellere Wiederherstellung der richteter Volumentherapie gezeigt [16]. Zudem weisen Daten einer Registeranalyse darauf hin, dass die Substitution von weniger als 3 I Vollelektrolytlösungen am OP-Tag einen positiven Effekt auf das 5-Jahres-Überleben nach onkologischer Kolorektalchirurgie zu haben scheint [2].

Trotz der guten Evidenz für die Wirksamkeit der FT-Maßnahmen erfolgt die Umsetzung nur sehr zögerlich. Neben fehlenden personellen und finanziellen Resgastrointestinalen Motilität bei zielgesourcen sowie dem Festhalten an etablier- ten, traditionellen Behandlungskonzepten ist die unzureichende Zusammenarbeit im interdisziplinären Team (Anästhesie, Chirurgie, Ernährungsmedizin, Pflege, Physiotherapie) einer der am häufigsten angeführten Gründe für das Scheitern [12].

Interessanterweise fokussieren sich trotz des interdisziplinären Ansatzes von FT-Programmen nahezu alle Studien maßgeblich auf die chirurgischen Aspekte bzw. deren Umsetzung. Ziel dieser deutschlandweiten Umfrage unter den Mitgliedern der Deutschen Gesellschaft für Anästhesie und Intensivmedizin (DGAl) war es daher, die Adhärenz hinsichtlich der interdisziplinären und anästhesiologischen Aspekte der FT-Maßnahmen zu evaluieren und zu analysieren, wo Optimierungsbedarf besteht.

\section{Untersuchungsmethoden}

Über das Online-Umfragetool SurveyMonkey von momentive.ai ${ }^{\circledR}$ (www.surveymon key.de) wurde ein Fragebogen zum anästhesiologischen Management bei kolorektalen Operationen über den Mail-Verteiler der DGAI an die Mitglieder verschickt (Zusatzmaterial online). Im Zeitraum vom 28.01.2021 bis zum 31.03.2021 konnte der Fragebogen online beantwortet werden. Die statistische Auswertung der Daten erfolgte mit Microsoft Excel (Microsoft Corporation).

\section{Ergebnisse}

Von 12.975 kontaktierten Mitgliedern der DGAI haben 435 Personen an der Umfrage teilgenommen (Rücklaufquote 3,4\%). Angaben zum Alter, zur beruflichen Position sowie zum Tätigkeitsbereich sind in - Tab. 1 angegeben.

\section{Umsetzung eines perioperativen FT-Behandlungskonzepts}

Zur Gewährleistung einer einheitlichen Patientenbetreuung sowie zur Durchsetzung klinikinterner Standards werden „standard operating procedures" (SOP) eingesetzt. $65,7 \%$ der Befragten $(n=286)$ bestätigten, dass an ihrer Klinik eine entsprechende SOP oder Vergleichbares zur perioperativen Patientenbetreuung bei kolorektalen Resektionen existiert. Die Umsetzung ei- 
Tab. 2 Übersicht einzelner Fast-Tack-Elemente und deren Umsetzung

Fast-Track-Empfehlung

\begin{tabular}{|l|l|}
\hline $\begin{array}{l}\text { Patientenaufklärung über das } \\
\text { Fast-Track-Konzept }\end{array}$ & $\begin{array}{l}\text { Adhä- } \\
\text { renz [\%] }\end{array}$ \\
50,0
\end{tabular}

Fast-Track-Konzept

Prähabilitation

\begin{tabular}{|l|l|}
\hline Empfehlung zur Nikotinkarenz & 21,6 \\
\hline
\end{tabular}

\begin{tabular}{|l|l|}
\hline Empfehlung zur Alkoholkarenz & 14,4
\end{tabular}

\begin{tabular}{|l|l|}
\hline Empfehlung zur Steigerung der & 23,7
\end{tabular}

körperlichen Aktivität

\begin{tabular}{l|l}
\hline Optimierung der Nahrungs- & 54,7
\end{tabular}

aufnahme/Vorbeugung von

Mangelernährung

\begin{tabular}{|l|l}
\hline Anämiescreening und Präventi- & 64,0
\end{tabular}

on im Sinne eines Patient Blood

Management

\begin{tabular}{l|l} 
Keine routinemäßige sedieren- & 65,4
\end{tabular}

de Prämedikation

Präoperative Nüchternheitsphase

\begin{tabular}{|l|l}
\hline Nahrungsaufnahme bis $6 \mathrm{~h}$ vor & 56,6
\end{tabular}

Operationsbeginn

Trinken klarer Flüssigkeiten bis $\quad 71,3$

$2 \mathrm{~h}$ vor der Operation

Perioperatives Wärmemanagement

\begin{tabular}{|l|l}
\hline Präoperativ während der Narko- & 58,1
\end{tabular}

seeinleitung

\begin{tabular}{l|l} 
Intraoperativ & 96,
\end{tabular}

\begin{tabular}{|l|l}
\hline Postoperativ im Aufwachraum & 65,3
\end{tabular}

$\begin{array}{ll}\text { Narkoseführung mittels totaler } & 16,4\end{array}$

intravenöser Anästhesie (TIVA)

\begin{tabular}{ll|l}
\hline Restriktives oder bedarfsadap- & 77,3
\end{tabular}

tiertes Volumenmanagement

\begin{tabular}{l|l} 
PONV-Prophylaxe & 90,3
\end{tabular}

Opioidsparende Analgesie (offen/MIC)

\begin{tabular}{|l|l}
\hline Einsatz von Periduralkathetern & $94,0 / 55,3$ \\
\hline
\end{tabular}

\begin{tabular}{|l|l|}
\hline Einsatz einer TAP-Blocks & $3,7 / 8,0$ \\
\hline Lokale Infiltration des Operati- & $18,4 / 26,7$
\end{tabular}

onszugangs

\begin{tabular}{l|l} 
Mobilisation an die Bettkante & 17,6
\end{tabular}

im Aufwachraum

\begin{tabular}{l|l}
\hline Anbieten von kohlenhydrat- & 7,3
\end{tabular}

haltigen Trinklösungen nach

Rückkehr der Schutzreflexe

PONV Postoperative nausea and vomiting,

MIC Minimalinvasive Chirurgie, TAP Transver-

sus abdominis plane Block

nes standardisierten FT-Behandlungskonzepts wird jedoch lediglich durch $43,7 \%$ der Teilnehmer $(n=190)$ angegeben. Die Existenz eines interdisziplinären FT-Teams, welches mindestens aus Kollegen der Chirurgie und Anästhesie besteht, wurde zudem von nur 128 Umfrageteilnehmern $(28,4 \%)$ berichtet.

Tab. 3 Prämedikation

Erhalten Ihre Patienten vor kolorektalen Eingriffen standardmäßig eine $\quad$ Prozent $(n=434)$ Prämedikation?

Ja, Benzodiazepine

$27,9(n=121)$

Ja, Clonidin

Nur auf Nachfrage des Patienten

$0,7(n=3)$

Nein

$34,8(n=151)$

$30,7(n=133)$

\begin{tabular}{|l|l} 
Sonstiges (Oxycodon, abhängig vom Alter, abhängig von Vorerkrankungen) & $6,0(n=26)$
\end{tabular}

Eine Gegenüberstellung einzelner FTElemente und deren Umsetzung gemäß der Umfrageergebnisse wird in DTab. 2 dargestellt.

\section{Präoperative Patienteninformation} und Prähabilitation

Über die Hälfte der Befragten gaben an, dass in ihrer Klinik keine spezielle Aufklärung über das perioperative Behandlungskonzept vor kolorektalen Eingriffen erfolgt. In Klinken, in denen eine derartige Aufklärung Teil der präoperativen Patientenvorbereitung ist, erfolgte diese nach Angabe der Teilnehmer in $84,7 \%$ der Fälle durch die Chirurgie oder eine Patientenbroschüre/ein Informationsvideo. Eine Aufklärung über das FT-Konzept im Rahmen der Vorstellung in der Anästhesieambulanz wird nur durch 33 Teilnehmer $(7,6 \%)$ durchgeführt.

Beratungen oder Schulungen zur Nikotin- oder zur Alkoholkarenz als Vorbereitung auf die geplante Operation werden in $13,8 \%(n=60)$ bzw. $9,2 \%(n=40)$ der Kliniken angeboten. Auch Empfehlungen zur Steigerung der körperlichen Aktivität werden nur gemäß $15,2 \%(n=66)$ der Befragten routinemäßig ausgesprochen. Maßnahmen zur Vorbeugung von Mangelernährung bzw. zur Optimierung der Nahrungsaufnahme werden zumindest in $34,9 \%(n=152)$ der Kliniken angeboten. Selbst die Durchführung eines präoperativen Anämiescreenings wird nur von 40,9\% $(n=178)$ der Befragten bestätigt.

\section{Prämedikation}

Während 27,9\% $(n=121)$ der Befragten angeben, dass eine sedierende Prämedikation mit Benzodiazepinen standardmäBig verschrieben wird, geben die meisten Umfrageteilnehmer an $(34,8 \%, n=151)$, dass eine Prämedikation nur auf Nachfra- ge des Patienten angeordnet wird. Weitere $30,7 \%(n=133)$ berichten, dass die Patienten keine standardmäßige Prämedikation erhalten ( $\bullet$ Tab.3). Sofern eine Prämedikation erfolgt, wird diese in $79,2 \%(n=248)$ am Morgen vor der Operation verabreicht. Lediglich $10 \%(n=31)$ verschreiben eine Prämedikationzur Einnahme am Vorabend und am Morgen vor der Operation, und nur $1,6 \%$ der Befragten $(n=7)$ geben an, die Prämedikation zur alleinigen Einnahme am Abend vor der Operation zu verordnen.

\section{Perioperatives Wärmemanagement}

Zur Verhinderung einer perioperativen Hypothermie beginnen 44,4\% $(n=192)$ bereits beim Eintreffen des Patienten im Wartebereich des OP mit aktiven Wärmemaßnahmen. Fast alle $(n=415)$ führen die Wärmemaßnahmen intraoperativ durch. Selbst postoperativ geben noch zwei Drittel der Befragten an, ihre Patienten $(65,3 \%$, $n=282$ ) im Aufwachraum aktiv zu wärmen. Nahezu alle Befragten verwenden hierbei Warmluftsysteme (• Tab. 4).

\section{Narkoseführung und intraoperative Analgesie}

$80 \%$ der Befragten $(n=341)$ verwenden zur Aufrechterhaltung der Narkose ein balanciertes Anästhesieverfahren, 16,4\% $(n=70)$ nutzen die totale intravenöse Anästhesie (TIVA) mit Propofol. Für die intraoperative Analgesie werden gemäß $94,2 \%$ der Teilnehmer $(n=407)$ v.a. i.v.Opioide eingesetzt. Doch auch die epidurale Anwendung von Opioiden kommt zu $62,3 \%(n=269)$ regelmäßig zum Einsatz. Zudem werden Lokalanästhetika epidural $(76,4 \% ; n=330)$ routinemäßig eingesetzt (• Tab. 5). 
Tab. 4 Methoden des perioperativen Wärmemanagements Maßnahmen des perioperativen Wärmemanagements

Einsatz von Warmluftsystemen

Verabreichung angewärmter Infusionen

Atemerwärmung durch „minimal flow"

Erhöhung der Saaltemperatur

Organisation: geringe Wartezeiten, Patient nicht zu früh abdecken etc.

Sonstiges (Wärmematten, vorgeheizte Decken etc.)

Tab. 5 Intraoperative Analgesie

Eingesetzte Analgetika

Opioidanalgetika, i.v.

Opioidanalgetika, epidural

Lokalanästhetika, epidural

Lidocain, i.v.

Nichtopioidanalgetika (Metamizol, Paracetamol, Parecoxib)

Sonstiges (Ketamin; Parecoxib; Bupivacain, epidural; Morphin, intrathekal etc.)

\begin{tabular}{|l|l|}
\hline Tab. 6 Methoden der PONV-Prophylaxe & Prozent $(n=411)$ \\
\hline Maßnahmen zur PONV-Prophylaxe & $41,1(n=169)$ \\
\hline TIVA statt balancierter Anästhesie & $35,3(n=145)$ \\
\hline Weitgehender Verzicht auf Opioide (multimodale Schmerztherapie) & $98,8(n=406)$ \\
\hline Medikamentöse Prophylaxe & $3,7(n=15)$ \\
\hline $\begin{array}{l}\text { Sonstiges (Droperidol, Verzicht auf Benzodiazepine, Akupunktur/ } \\
\text { Akupressur) }\end{array}$ & \\
\hline PONV Postoperative nausea and vomiting & \\
\hline
\end{tabular}

\begin{tabular}{|l|l|l|}
\hline Tab. 7 Methoden der postoperativen Schmerztherapie \\
\hline $\begin{array}{l}\text { Häufig verwendete Maßnahmen zur post- } \\
\text { operativen Schmerztherapie }\end{array}$ & $\begin{array}{l}\text { Offene Operationen } \\
{[\%](n=430)}\end{array}$ & $\begin{array}{l}\text { Minimal-invasive } \\
\text { Operationen } \\
{[\%](n=430)}\end{array}$ \\
\hline PDK & $93,9(n=403)$ & $55,3(n=230)$ \\
\hline TAP-Block & $3,7(n=13)$ & $8,0(n=28)$ \\
\hline Lokale Infiltration des Operationszugangs & $18,4(n=65)$ & $26,7(n=95)$ \\
\hline Schmerzpumpe & $50,4(n=195)$ & $36,0(n=136)$ \\
\hline Kurzinfusionen/orale Gabe von Opioiden & $71,8(n=282)$ & $74,9(n=292)$ \\
\hline $\begin{array}{l}\text { Alleinige Analgesie mittels Nichtopioidanal- } \\
\text { getika }\end{array}$ & $15,1(n=54)$ & $18,2(n=64)$ \\
\hline PDK Periduralkatheter, TAP Transversus abdominis plane Block & \\
\hline
\end{tabular}

\section{Volumenmanagement}

Bezüglich der perioperativen Volumentherapie wird durch die meisten Befragten angegeben, ein restriktives Volumenmanagement zu bevorzugen (72,4\%; $n=312) .18,3 \%(n=79)$ geben jedoch an, dass ein liberales Volumenmanagement an ihrer Klinik standardmäßig durchgeführt wird. Eine zielgerichtete Volumentherapie (",goal directed") erfolgt nur in 4,9\% $(n=21)$ der Kliniken. Unabhängig davon,
PONV-Prophylaxe

Zur Vermeidung postoperativer Übelkeit und Erbrechen (PONV) geben 45,6\% der Befragten $(n=197)$ an, bei jeder Darmresektion eine PONV-Prophylaxe durchzuführen. Weitere $44,7 \%(n=193)$ berichten, eine PONV-Prophylaxe nur bei Vorliegen von Risikofaktoren zu veranlassen. Lediglich $8,1 \%(n=35)$ berichten, bei elektiven kolorektalen Eingriffen keine derartige Therapie einzuleiten. Das hierbei mit großem Abstand am häufigsten angewendete Verfahren ist die medikamentöse Prophylaxe (•Tab.6).

\section{Postoperative Analgesie}

Eine postoperative analgetische Therapie mit i.v.- oder oralen Opioiden ist sowohl bei offenen Operationen $(71,8 \% ; n=282)$ als auch bei minimal-invasiven Eingriffen (MIC) $(74,8 \% ; n=292)$ weit verbreitet. Eine andere, v. a. nach offenen Operationen eingesetzte Methode ist die Applikation von Lokalanästhetika über einen Periduralkatheter (PDK) $(93,9 \% ; n=403)$. Doch auch nach MIC wird dieser gemäß 55,3\% der Befragten $(n=230)$ regelmäßig verwendet. Lokale Infiltrationen des Operationszugangs oder eine lokale Nervenblockade (z. B. mittels „Transversus-abdominisplane"(TAP)-Block) kommen unabhängig vom Zugangsweg nur selten zum Einsatz (•Tab. 7).

Interdisziplinäres FT-Team und Zusammenarbeit mit der Chirurgie im Bereich der perioperativen Medizin

Die Zusammenarbeit mit den chirurgischen Kollegen wurde im Durchschnitt als "befriedigend" bewertet (Mittelwert 2,9; $\mathrm{SD} \pm 1,2$ ) (Zusatzmaterial online). Allerdings bewerten ein Drittel der Befragten die Zusammenarbeit mit der Schulnote 4 oder schlechter.

\section{Diskussion} lauf auf $1-3 \mid$ geschätzt. Hierbei handelt es sich ausschließlich $(99,8 \% ; n=431)$ um kristalloide Lösungen.
Bereits 2016 veröffentlichte die ERAS Society ein Consensus-Statement mit 18 Empfehlungen zur optimalen perioperativen anästhesiologischen Betreuung von viszeralchirurgischen Patienten [3]. Diese Empfehlungen beruhen auf Daten 
aus verschiedenen Studien, die zeigen konnten, dass die stringente Umsetzung der anästhesiologischen Bausteine des FT-Konzepts (z.B. Prämedikation, Volumenmanagement, Wärmemanagement, intra- und postoperative Schmerztherapie etc.) direkt mit der Komplikationsrate und der postoperativen KVD korrelieren [4]. Dies bedeutet, dass mit einer Steigerung der Adhärenz zu den FT-Maßnahmen die Komplikationsrate sinkt [4]. Mittlerweile gibt es gute Hinweise, dass die Umsetzung einzelner intraoperativer Bausteine (v.a. Volumenmanagement) nicht nur einen positiven Effekt auf das kurzfristige Outcome hat, sondern auch das 5-JahresÜberleben nach onkologischer Kolorektalchirurgie verbessert wird [2]. Dies macht deutlich, dass eine konsequente, fachübergreifende Umsetzung der einzelnen FTBausteine wie auch des Gesamtkonzepts alternativlos ist.

Inwieweit das perioperative FT-Konzept in deutschen Kliniken allerdings verbreitet ist, und wie dessen Umsetzung erfolgt, verbleibt aktuell unklar. Um den Status quo hinsichtlich der Adhärenz zu den anästhesiologischen Maßnahmen zu erheben, führten wir eine Umfrage unter den Mitgliedern derDGAI durch. Im Anschluss wurden die Ergebnisse der Umfrage mit den aktuellen Empfehlungen verglichen und analysiert, wo Optimierungsbedarf besteht.

Wir untersuchten zunächst, welche Strukturen zur Umsetzung eines FT-Konzepts in den anästhesiologischen Abteilungen existieren. Studien konnten hierbei zeigen, dass insbesondere das Vorhandensein von SOP ein wesentlicher Faktor für eine erfolgreiche Implementierung und Aufrechterhaltung der FTMaßnahmen ist [12]. Gemäß der Umfrage existiert in weniger als der Hälfte der Kliniken eine entsprechende SOP. Ohne Standardisierung wird eine einheitliche Patientenbetreuung erschwert, was in höherem Aufwand und Mehrarbeit resultiert. Dies wiederum senkt die Motivation und das Engagement der beteiligten Mitarbeiter $[12,14]$. Neben dem Engagement der Mitarbeiter ist eine spezifische Aufklärung der Patienten über das FT-Konzept wichtig. Hier liegt gemäß der Umfrage ebenfalls Optimierungsbedarf: Entweder es erfolgt keine Aufklärung, oder die Zuständig- keiten, welche Abteilung über welche Maßnahmen aufklärt, ist unklar. Eine klare Aufgabenzuweisung könnte bereits zu einer Verbesserung der Gesamtsituation führen. Insbesondere in Zeiten mit reduzierten zeitlichen und personellen Ressourcen wären gemeinsame OnlineTutorials, Videos oder interdisziplinär verfasste Broschüren eine hervorragende Alternative, wie Untersuchungen zeigen konnten [1]. So wird das Bewusstsein der Patienten für die Sinnhaftigkeit der Maßnahmen geschärft, und die Compliance sowie die Patientenzufriedenheit steigen.

Zu den Aufklärungen im weiteren Sinne gehören auch Beratungsgespräche bezüglich der Sinnhaftigkeit von körperlicher Aktivität, Alkohol- und Nikotinkarenz sowie das Anämie- und Malnutritionsscreening. Für all diese sog. Prähabilitationsmaßnahmen konnte in verschiedenen Metaanalysen eine Reduktion der Morbidität und der Mortalität sowie eine verkürzte KVD nachgewiesen werden [13]. Die Evidenz ist hier so gut, dass mittlerweile Operationen zur präoperativen Optimierung verschoben werden [13]. Dennoch zeigen die von uns erhobenen Daten, dass die Empfehlungen in Deutschland unzureichend umgesetzt werden. Während beispielsweise die ERAS Society eine individuelle Beratung und pharmakologische Unterstützung zur präoperativen Nikotin- und Alkoholabstinenz empfiehlt [6], werden nur wenige Patienten (13,8\% bzw. 9,2\%) über die Sinnhaftigkeit einer mindestens 4-wöchigen präoperativen Karenz aufgeklärt oder Maßnahmen ergriffen. Auch Empfehlungen zur Steigerung der körperlichen Aktivität vor einer geplanten Operation werden trotz eindeutiger Daten zu selten ausgesprochen. Weiterhin wird die präoperative Verwendung von Scoring-Tools in der Literatur empfohlen, um Patienten mit erhöhtem Risiko für perioperative Komplikationen zu identifizieren [9]. Unsere Umfrage zeigt jedoch, dass selbst Screenings auf Mangelernährung oder das Vorliegen einer Anämie in Deutschland in weniger als der Hälfte der Fälle standardmäßig durchgeführt werden. Da sowohl Mangelernährung als auch Anämien insbesondere bei onkologischen Patienten häufig vorkommen und großen Einfluss auf die Komplikationsrate sowie die onkologische Prognose haben, ist die geringe Umsetzung insbesondere im Hinblick auf die eindeutige Evidenz nicht nachvollziehbar. Über die Ursache kann nur spekuliert werden: Am ehesten sind jedoch erneut unklare $\mathrm{Zu}$ ständigkeiten, fehlende zeitliche und personelle Ressourcen sowie eine unklare Kostenübernahme und andere wirtschaftliche Aspekte zu nennen. Durch den Aufbau eines interdisziplinären FT-Teams könnte dieses Problem ohne großen Aufwand gelöst werden.

Psychischer Stress vor einer Operation kann zu einer erhöhten postoperativen Schmerzwahrnehmung und einer höheren Komplikationsrate führen [8]. Aus diesem Grunde wurde über Jahre eine Prämedikation zur Anxiolyse verabreicht. Studien haben jedoch gezeigt, dass es hierdurch zu protrahierten postoperativen Verläufen und der erhöhten Inzidenz eines Delirs kommt [6]. Daher wird aktuell eine Patientenaufklärung zur Stressreduktion mit Verzicht auf die routinemäßige Anwendung sedierender Medikamente, insbesondere bei älteren Patienten, empfohlen. Der Alltag in deutschen Kliniken ist allerdings gemäß der Umfrage ein anderer: Ein Drittel der Befragten verwendet regelhaft eine sedierende Prämedikation. Nur etwa 30\% verwenden keine Medikamente und handeln somit gemäß der Empfehlung. Unklar verbleibt, was den Patienten anstelle dessen zur Stressreduktion angeboten wird. Insbesondere das Erlernen stressreduzierender Coping-Strategien als Alternative ist zeitlich aufwendig und daher im Alltag nur schwer umsetzbar. Ein gangbarer Mittelweg wäre beispielsweise die selektive Anordnung von kurz wirksamen Benzodiazepinen bei Patienten, die im Rahmen einer Evaluierung mittels der Amsterdam Preoperative Anxiety and Information Scale (APAIS) einen erhöhten präoperativen Stress- oder Angstlevel haben [15].

Die intraoperative sowie unmittelbar postoperative i.v.-Flüssigkeitssubstitution hat für den weiteren Verlauf entscheidende Bedeutung. So führt eine Volumenüberladung nach Abdominalchirurgie beispielsweise zu einer erhöhten Komplikationsrate und einem schlechteren onkologischen Outcome [16]. Eine restriktive oder bedarfsadaptierte/zielgerichtete Flüssigkeitssubstitution hingegen verkürzt die KVD und reduziert die Rate an infektiologischen Komplikationen. „Restriktiv" 
bedeutet jedoch keine Unterversorgung mit Volumen, sondern zielt auf eine ausgeglichene Bilanzierung („zero balance approach") ab. Bei Risikopatienten oder komplexen Operationen sollte zusätzlich der Einsatz von erweitertem hämodynamischen Monitoring und "goal directed therapy" erfolgen [6].

Die Mehrheit der Umfrageteilnehmer (75\%) gab eine Präferenz für ein restriktives Volumenmanagement, was am ehesten einer intraoperativen Flüssigkeitssubstitution von 1-3 I entspricht. Dies beinhaltet jedoch nicht die Menge an Infusionen, die postoperativ im Aufwachraum und anschließend auf der Station verabreicht wird, woraus letztlich eine höhere Menge an infundiertem Volumen resultiert. Da sich mit dem Abschluss der chirurgischen Maßnahmen und dem Ende der Narkose auch der Flüssigkeitsbedarf ändert und der Patient mit dem Wiederkehren der Schutzreflexe wieder trinken kann, sollte im weiteren Verlauf die i.v.-Flüssigkeitsgabe zurückhaltend erfolgen und dem Patienten bereits im Aufwachraum Trinknahrung angeboten werden. Dies sollte dann im weiteren Verlauf auf der Station fortgeführt und die i.v.Substitution rasch beendet werden. Hierfür ist eine gute Kommunikation zwischen den Schnittstellen OP, Aufwachraum und Station notwendig. Zudem sollte darauf geachtet werden, dass die Patienten zu Beginn der Operation über einen ausgeglichenen Flüssigkeitshaushalt verfügen. Hierfür sollten zu lange Nüchternheitsphasen vermieden werden und dem Patienten bis $2 \mathrm{~h}$ vor der Operation kohlenhydratreiche Trinklösungen angeboten werden [6].

PONV gehören zu den häufigsten Komplikationen nach chirurgischen Eingriffen, durch verschiedene Maßnahmen kann die Inzidenz jedoch unter $10 \%$ gesenkt werden [11]. Aus diesem Grund ist die medikamentöse PONV-Prophylaxe ein integraler Bestandteil des FT-Konzepts und wird flächendeckend eingesetzt, wie unsere Umfrage zeigt. Weitere Maßnahmen, wie der Einsatz einer TIVA, können das PONV-Risiko ebenfalls deutlich reduzieren und werden als Bestandteile des FTKonzepts empfohlen [4]. In Deutschland wird laut unserer Umfrage die Narkoseaufrechterhaltung überwiegend mit volatilen Anästhetika durchgeführt. Dies lässt sich mit geringeren Kosten, einer erhöhten Sicherheit durch die exspiratorische Gasmessung und einer besseren Steuerbarkeit, v.a. bei längerer Anwendungsdauer, verglichen mit einer TIVA, erklären [7]. Mit der Zunahme von zur Verfügung stehendem prozessierten EEG-Monitoring sowie der Anwendung von „Target-controlled-infusion"-Modellen lassen sich jedoch diese Nachteile gut kompensieren.

Eine wichtige Komponente im Rahmen des FT-Konzepts ist die intra- und postoperative Schmerztherapie. Als PONV-Prophylaxe und zur Verhinderung von Nebenwirkungen (Schwindel, Obstipation etc.) sollte diese unter Einsparung von Opioiden erfolgen. Gemäß der Umfrage sind Opioide jedoch die häufigste Wahl zu intraoperativer und postoperativer Schmerztherapie. Das Verfahren der Wahl ist jedoch insbesondere in der offenen Chirurgie der PDK: Durch die kontinuierliche, bereits intraoperativ begonnene Applikation von Lokalanästhetika können intra- und postoperativ Opioide eingespart werden. Die zusätzliche Sympathikolyse führt zudem zu einer rascheren Erholung der postoperativen Darmfunktion. Deshalb stellt der PDK weiterhin den Goldstandard in der postoperativen Schmerztherapie dar. Bei MIC sind hingegen die Vorteile aufgrund des minimal-invasiven Zugangs und der geringen Darmmanipulation intraoperativ nur noch marginal; eine Verkürzung der KVD kann im Gegensatz zur offenen Chirurgie nicht erreicht werden. Hier empfehlen verschiedene FT-Protokolle die i.v.Verabreichung von Lidocain [6]. Dies konnte jedoch in Deutschland trotz guter Evidenz bisher noch nicht großflächig etabliert werden. Weitere, sehr elegante Optionen zur postoperativen Analgesie, insbesondere bei MIC, sind periphere Regionalanästhesieverfahren, wie die Anlage eines TAP-Blockes. Die analgetische Wirkung hält bis zu $24 \mathrm{~h}$, und die Patienten haben keine zusätzliche Leitung, die eine Mobilisation erschweren könnte. Vergleichende Studien mit einem PDK oder einer Schmerzpumpe zeigen Vorteile für den TAP-Block hinsichtlich der KHV bei gleichwertiger analgetischer Wirksamkeit und unverändert positivem Effekt auf die Funktion des GI-Trakts [17]. Allerdings wird dieses noch recht neue Verfahren in Deutschland noch nicht flächendeckend eingesetzt. Die Gründe hier- für liegen vermutlich in der fehlenden Erfahrung, evtl. auch am Festhalten tradierter perioperativer Konzepte und der Skepsis des Operateurs gegenüber dem neuen Verfahren.

Bei der perioperativen Medizin handelt es sich um ein interdisziplinäres Fach. Nahezu alle Studien zur erfolgreichen Implementierung des FT-Programms in Kliniken benennen daher eine gute interdisziplinäre Zusammenarbeit und den Aufbau eines interdisziplinären Teams als wichtigste Grundvoraussetzung [12]. Nur 29,4\% der Befragten gaben jedoch an, dass an ihrem Krankenhaus solch ein interdisziplinäres Team für die Umsetzung eines FT-Konzepts sowie dessen Aufrechterhaltung verantwortlich ist. Weiterhin wird von mehr als einem Drittel der Befragten die interdisziplinäre Zusammenarbeit mit der Note "ausreichend" oder schlechter bewertet. Korreliert man allerdings die Angaben der Befragten zum Vorhandensein eines FT-Teams und die Bewertung der interdisziplinären Zusammenarbeit, so zeigt sich, dass 85,1\% der Anästhesisten, die in einem Krankenhaus mit einem etablierten FT-Team arbeiten, mit der interdisziplinären Zusammenarbeit zufrieden sind (Noten 1-3), während in Krankenhäusern, in denen kein solches Team existiert, nur $60,6 \%$ die Zusammenarbeit als befriedigend oder besser bewerten. Dieses Ergebnis ist ein eindeutiges Plädoyer für die Etablierung interdisziplinärer Teams. Neben ärztlichen Kollegen verschiedener Fachrichtungen sind Pflegekräfte, Physiotherapeuten und insbesondere sog. FastTrack-Assistenten wichtige Bestandteile eines solchen Teams. Diese speziell geschulten Pflegekräfte sollen als Bindeglied zwischen Patienten, Pflegepersonal und Ärzten fungieren und das reguläre Stationsteam entlasten, indem sie die Patienten motivieren und z. B. bei der Mobilisierung unterstützen. Gleichzeitig sollen sie die rechtzeitige Durchführung wichtiger Interventionen im Auge behalten und die Behandelnden bei Bedarf daran erinnern [12].

Ein weiterer Aspekt, der die Umsetzung des FT-Konzepts in Zukunft fördern und erleichtern soll, ist eine derzeit in Erarbeitung befindliche S3-Leitlinie zur perioperativen Therapie von gastrointestinalen Tumoren, die als einheitlicher Leitfaden zur Behand- 
lung gemäß des FT-Konzepts dienen soll [14].

\section{Limitationen}

Zu den Limitationen unserer Erhebung gehören neben dem kaum vermeidbaren „,social bias" der "non response bias". Bei einer Rücklaufquote von gerade einmal 3,4\% muss dies berücksichtigt werden. Die Ursache für die geringe Teilnahme ist für uns unklar, deckt sich leider aber mit einer ähnlichen Umfrage unter Viszeralchirurgen. Da jedoch alle Ausbildungsstufen sowie verschiedene Versorgungseinrichtungen vertreten waren, sind wir der Meinung, dass das Teilnehmerkollektiv dennoch als repräsentativ angesehen werden kann.

\section{Fazit für die Praxis}

- Trotz guter Evidenz ist die Umsetzung der anästhesiologischen Fast-Track(FT)Bausteine in Deutschland verbesserungswürdig.

- Dies betrifft insbesondere interdisziplinäre Bausteine (Prähabilitation) sowie Maßnahmen an den Schnittstellen OP, Aufwachraum und Station (perioperatives Volumenmanagement, intra- und postoperative Analgesie).

- Ursachen für die unzureichende Umsetzung liegen vermutlich in unklaren $\mathrm{Zu}$ ständigkeiten der beteiligten Fachbereiche und optimierbarer interdisziplinärer Kommunikation.

- Für eine höhere Adhärenz ist die Etablierung eines interdisziplinären FT-Teams die entscheidende Maßnahme.

- In einem zweiten Schritt sollte das interdisziplinäre Team SOP zum FT Konzept verfassen, diese regelmäßig überprüfen und gemeinsam überarbeiten. Durch diese verbesserten Strukturen sinkt der Arbeitsaufwand, was wiederum die Motivation der Mitarbeiter verbessert und zeitliche Ressourcen schafft.
Korrespondenzadresse

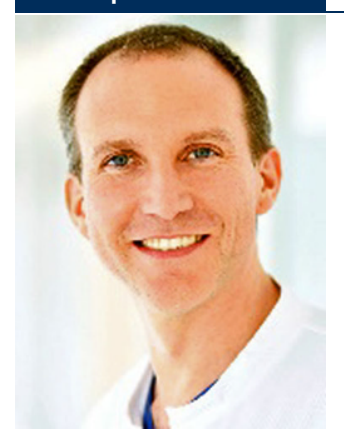

PD Dr. med. T. O. Vilz

Klinik- und Poliklinik für Allgemein-, Viszeral-, Thorax- und Gefäßchirurgie, Universitätsklinikum Bonn

Venusberg-Campus 1, 53127 Bonn, Deutschland tim.vilz@ukbonn.de

Danksagung. Die Autoren möchten sich bei der Deutschen Gesellschaft für Anästhesiologie und Intensivmedizin (DGAl) für ihre Unterstützung bedanken!

Funding. Open Access funding enabled and organized by Projekt DEAL.

\section{Einhaltung ethischer Richtlinien}

Interessenkonflikt. M. A. Willis, M. Thudium, C. J. van Beekum, M. Söhle, M. Coburn, J. C. Kalff und T. O. Vilz geben an, dass kein Interessenkonflikt besteht.

Für diesen Beitrag wurden von den Autoren keine Studien an Menschen oder Tieren durchgeführt. Für die aufgeführten Studien gelten die jeweils dort angegebenen ethischen Richtlinien.

Open Access. Dieser Artikel wird unter der Creative Commons Namensnennung 4.0 International Lizenz veröffentlicht, welche die Nutzung, Vervielfältigung, Bearbeitung, Verbreitung und Wiedergabe in jeglichem Medium und Format erlaubt, sofern Sie den/die ursprünglichen Autor(en) und die Quelle ordnungsgemäß nennen, einen Link zur Creative Commons Lizenz beifügen und angeben, ob Änderungen vorgenommen wurden.

Die in diesem Artikel enthaltenen Bilder und sonstiges Drittmaterial unterliegen ebenfalls der genannten Creative Commons Lizenz, sofern sich aus der Abbildungslegende nichts anderes ergibt. Sofern das betreffende Material nicht unter der genannten Creative Commons Lizenz steht und die betreffende Handlung nicht nach gesetzlichen Vorschriften erlaubt ist, ist für die oben aufgeführten Weiterverwendungen des $\mathrm{Ma}$ terials die Einwilligung des jeweiligen Rechteinhabers einzuholen.

Weitere Details zur Lizenz entnehmen Sie bitte der Lizenzinformation auf http://creativecommons.org/ licenses/by/4.0/deed.de.

\section{Literatur}

1. Adams KT, Walker AD, Searson E et al (2017) Improving patient satisfaction using a video-based patient education platform, S217-224

2. Asklid D, Segelman J, Gedda C et al (2017) The impact of perioperative fluid therapy on short-term outcomes and 5-year survival among patients undergoing colorectal cancer surgery-A prospective cohort study within an ERAS protocol. Eur JSurg Oncol 43(8):1433-1439

3. Feldheiser A, Aziz O, BaldiniGetal (2016) Enhanced Recovery After Surgery (ERAS) for gastrointestinal surgery, part 2: consensus statement for anaesthesia practice. Acta Anaesthesiol Scand 60(3):289-334

4. Grant MC, Pio Roda CM, Canner JK et al (2019) The impact of anesthesia-influenced process measure compliance on length of stay: results from an enhanced recovery after surgery for colorectal surgery cohort. Anesth Analg 128(1):68-74

5. Gustafsson UO, Oppelstrup H, Thorell A et al (2016) Adherence to the ERAS protocol is associated with 5-year survival after colorectal cancer surgery: a retrospective cohort study. World J Surg 40(7):1741-1747

6. Gustafsson UO, Scott MJ, Hubner M et al (2019) Guidelines for perioperative care in elective colorectal surgery: enhanced recovery after surgery $\left(\right.$ ERAS $\left.^{\circledR}\right)$ society recommendations: 2018. World J Surg 43(3):659-695

7. Hoetzel A (2019) Klinischer Stellenwert von total intravenöser Anästhesie (TIVA) und Inhalationsanästhesie Inhalationsanästhesie. Anasth Intensivmed. https://doi.org/10.19224/ai2019. 174

8. Mavros MN, Athanasiou S, Gkegkes ID et al (2011) Do psychological variables affect early surgical recovery? Plos One. https://doi.org/10. 1371/journal.pone.0020306

9. National Institute for Health and Care Excellence (UK) (2020) Perioperative care in adults. National Institute for Health and Care Excellence (UK), London

10. Pisarska M, Pędziwiatr M, Małczak P et al (2016) Do we really need the full compliance with ERAS protocol in laparoscopic colorectal surgery? A prospective cohort study. Int J Surg 36(Pt A):377-382

11. Rüsch D, Eberhart LHJ, Wallenborn J et al (2010) Nausea and vomiting after surgery under general anesthesia: an evidence-based review concerning risk assessment, prevention, and treatment. Dtsch Arztebl Int 107(42):733-741

12. van Beekum C, Stoffels B, von Websky Met al (2020) Implementierung eines Fast-Track-Programmes: Herausforderungen und Lösungsansätze. Chirurg 91(2):143-149

13. Waterland JL, McCourt O, Edbrooke L et al (2021) Efficacy of prehabilitation including exercise on postoperative outcomes following abdominal cancer surgery: a systematic review and metaanalysis. Front Surg. https://doi.org/10.3389/fsurg. 2021.628848

14. Willis MA, Schwenk W, Post S et al (2021) Hintergrund, Notwendigkeit und Methodik der S3-Leitlinie "Perioperatives Management bei gastrointestinalen Tumoren (POMGAT)“. Zentralbl Chir 146(3):241-248

15. Work Group of the CPG on Perioperative Care in Major Abdominal Surgery (2016) Clinical practice guideline on perioperative care in major abdominal surgery 
16. Wrzosek A, Jakowicka-Wordliczek J, Zajaczkowska $R$ et al (2019) Perioperative restrictive versus goal-directed fluid therapy for adults undergoing major non-cardiac surgery. Cochrane Database Syst Rev. https://doi.org/10.1002/14651858. CD012767.pub2

17. Xu YJ, Sun X, Jiang H et al (2020) Randomized clinical trial of continuous transversus abdominis plane block, epidural or patient-controlled analgesia for patients undergoing laparoscopic colorectal cancer surgery. Br JSurg 107(2):133-141

\section{Implementation of fast-track measures in colorectal resections. A survey among members of the DGAI}

Purpose: The fast-track (FT) concept is a multimodal, interdisciplinary approach to perioperative patient care intended to reduce postoperative complications. Despite good evidence implementation seems to need improvement, whereby almost all studies focused on the implementation of surgical modules regardless of the interdisciplinary aspect. Adherence to the anesthesiological measures (prehabilitation, premedication, volume and temperature management, pain therapy), on the other hand, has been insufficiently studied. To assess the status quo a survey on the implementation of anesthesiological FT measures was conducted among members of the German Society of Anesthesiology and Intensive Care Medicine (DGAl) to analyze where potential for improvement exists.

Methods: Using the SurveyMonkey ${ }^{\circledR}$ online survey tool, 28 questions regarding perioperative anesthesiological care of colorectal surgery patients were sent to DGAI members in order to analyze adherence to FT measures.

Results: While some of the FT measures (temperature management, PONV prophylaxis) are already routinely used, there is a divergence between current recommendations and clinical implementation for other components. In addition to premedication, interdisciplinary measures (prehabilitation) and measures that affect multiple interfaces (operating theatre, recovery room, ward), such as volume management or perioperative pain management, are particularly affected.

Conclusion: The anesthesiological recommendations of the FT concept are only partially implemented in Germany. This particularly affects the interdisciplinary components as well as measures at the operating theatre, recovery room and ward interfaces. The establishment of an interdisciplinary FT team and interdisciplinary development of SOPs can optimize adherence, which in turn improves the short-term and long-term outcome of patients.

\section{Keywords}

Health services research $\cdot$ ERAS Perioperative care $\cdot$ Evidence-based medicine $\cdot$ Compliance 